Review

\title{
Circular RNA in Lung Cancer Research: Biogenesis, Functions, and Roles
}

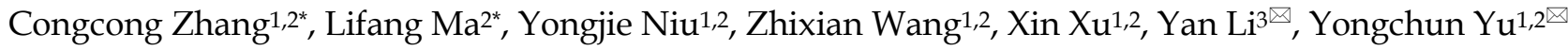 \\ 1. Shanghai Municipal Hospital of Traditional Chinese Medicine, Shanghai University of Traditional Chinese Medicine, Shanghai, 200071, P.R. China. \\ 2. Institute for Thoracic Oncology, Shanghai Chest Hospital, Shanghai Jiao Tong University, Shanghai, 200030, P.R. China. \\ 3. Department of Oncology, Shanghai Municipal Hospital of Traditional Chinese Medicine, Shanghai University of Traditional Chinese Medicine, \\ Shanghai,200071, P.R. China. \\ * These authors contributed equally to this study.
}

$\square$ Corresponding author: Prof. Yongchun Yu. No.241 West Huaihai Road, Xuhui District, Shanghai, P.R. China, 200030. Fax and Tel: 86-21-66313245, E-mail: yyc2166@sjtu.edu.cn; Prof. Yan Li. No.274 Middle Zhijiang Road, Jingan District, Shanghai, P.R. China, 200071.Fax and Tel: 86-21-63925588, E-mail: yan.xiaotian@shutcm.edu.cn

(1) The author(s). This is an open access article distributed under the terms of the Creative Commons Attribution License (https://creativecommons.org/licenses/by/4.0/). See http://ivyspring.com/terms for full terms and conditions.

Received: 2019.08.13; Accepted: 2019.12.18; Published: 2020.01.16

\begin{abstract}
Lung cancer is one of the most common and deadly malignancies worldwide, in spite of advances in targeted therapy in recent years. An effective strategy for lung cancer prevention remains a major problem. Advances in next-generation sequencing have helped in understanding the RNA and identifying novel circular RNAs (circRNAs) that may have a broad impact on the early diagnosis and treatment of lung cancer. The circRNAs, exhibiting spatiotemporal-specific expression, are stable and conserved and present diverse biological functions in the normal and diseased states, including cancer. In this review, we summarize the recent advances in elucidating the functional role of circRNAs in lung cancer pathogenesis and discuss their potential mechanisms.
\end{abstract}

Key words: Lung cancer, circRNA, diagnostic biomarker, therapeutic targets

\section{Introduction}

Lung cancer (LC) is the main cause of cancer mortality for both sexes globally. In 2018, 2.1 million new LC cases and 1.8 million deaths were reported worldwide, making LC the leading form of cancer in terms of both morbidity and mortality[1, 2]. Lung cancer has been pathologically classified into two main subsets: small cell lung cancer (SCLC), comprising $15 \%$ of the total cases[3], and the rest $85 \%$ non-small cell lung cancer (NSCLC)[4]. The NSCLC is classified into three subtypes; large cell lung carcinoma (LCLC; 10\%), lung squamous cell carcinoma (LUSC; 25\%), and lung adenocarcinoma (LUAD; 40\%). Recent advances in diagnostic and therapeutic procedures have resulted in a steady increase in the survival in most cancers, but not in LC whose 5-year survival rate is merely $17.7 \%$ [5]. The survival rate is considerably increased in patients with early-stage LC compared to patients with advanced LC. Therefore, it is crucial to establish the molecular mechanism underlying LC tumorigenesis and identify effective diagnostic biomarkers and therapeutic targets for LC.

The circRNAs are a class of covalently closed circular RNAs and ubiquitously exist in many species[6]. As a recently discovered form of endogenous non-coding RNA (ncRNA), circRNAs are typically generated from one to five exons [7] and are mainly located in the cytoplasm, with a small proportion of intron-containing circRNAs originating in the nucleus. Unlike linear RNAs, circRNAs exhibit the remarkable characteristic of being covalently closed continuous loop structure without $5^{\prime}$ to $3^{\prime}$ polarity and a polyadenylated tail, which makes them more stable in tissues and plasma[8]. Their unique structural properties suggest that these molecules are important transcripts, both physiologically and pathologically. Increasing studies have shown that circRNAs are expressed abnormally and act as an 
important regulatory element in the carcinogenesis and progression of LC, demonstrating that circRNAs can provide potential targets for the diagnosis and prognosis of LC. The present review briefly introduces current studies on the characteristics and biogenesis of circRNAs, summarizes their functional role and corresponding mechanisms in LC, and discusses the potential of circRNAs in the diagnosis and treatment.

\section{History of circRNAs}

In the 1970s[9], Sanger et al. initially identified circRNAs in RNA viruses, like plant viruses. For a long time, due to the limitations of the detection techniques, most circRNAs were considered as the products of mis-splicing or by-products of pre-mRNA processing. In 2012, the abundance and ubiquity of circRNA in eukaryotes was recognized due to advances in RNA high-throughput sequencing and bioinformatics.

\section{Biogenesis of circRNAs}

Based on the origin, circRNAs can be classified into three categories: exonic circRNAs (ecircRNAs), circular intron RNAs (ciRNAs), and exon-intron circRNAs (EIciRNAs) [10]. The biogenesis of these circRNAs is regulated by different circularization mechanisms (Figure 1). Among these, ecircRNAs are the most abundant type, accounting for the majority of identified circRNAs[11]. Three main models of ecircRNA formation have been reported, namely, lariat-driven, intron-pairing-driven, and RNAbinding proteins(RBPs)-driven circularization. These models can be divided into two mechanisms, direct back-splicing and exon skipping. In the lariat-driven circularization model, a downstream 50 splice site of an exon joins an upstream 30 splice site, resulting in exon-skipping and the formation of an RNA lariat consisting of several exons and introns. The introns are then removed to generate ecircRNA[12]. In some cases, exons are circularized with introns that are retained between the exons, resulting in the generation of EIciRNAs. In addition, intron-pairingdriven and RBP-driven circularization events are direct back-splicing mechanisms. During this process, two flanking introns bracketing circularized exons are brought into close proximity by repeated ALU complementary elements, other complementary sequences without repetition, or RBPs[13-15]. Circular intron RNA biogenesis, which occurs via a lariat-derived mechanism, mainly depends on the conserved motifs at both ends of the intron, i.e., a 7-nt GU-rich element near the $5^{\prime}$ splice site and an 11-nt C-rich element near the branch-point site[16].These motifs can prevent the intron from forming a circular debranching and cause the intron to form a circular structure. The resulting RNA circle is covalently linked through a $2^{\prime}, 5^{\prime}$-phosphodiester bond at the junction site. The $3^{\prime}$ tail, stretching from the $3^{\prime}$ end of the intron to the branch point, is removed to produce stable ciRNAs.

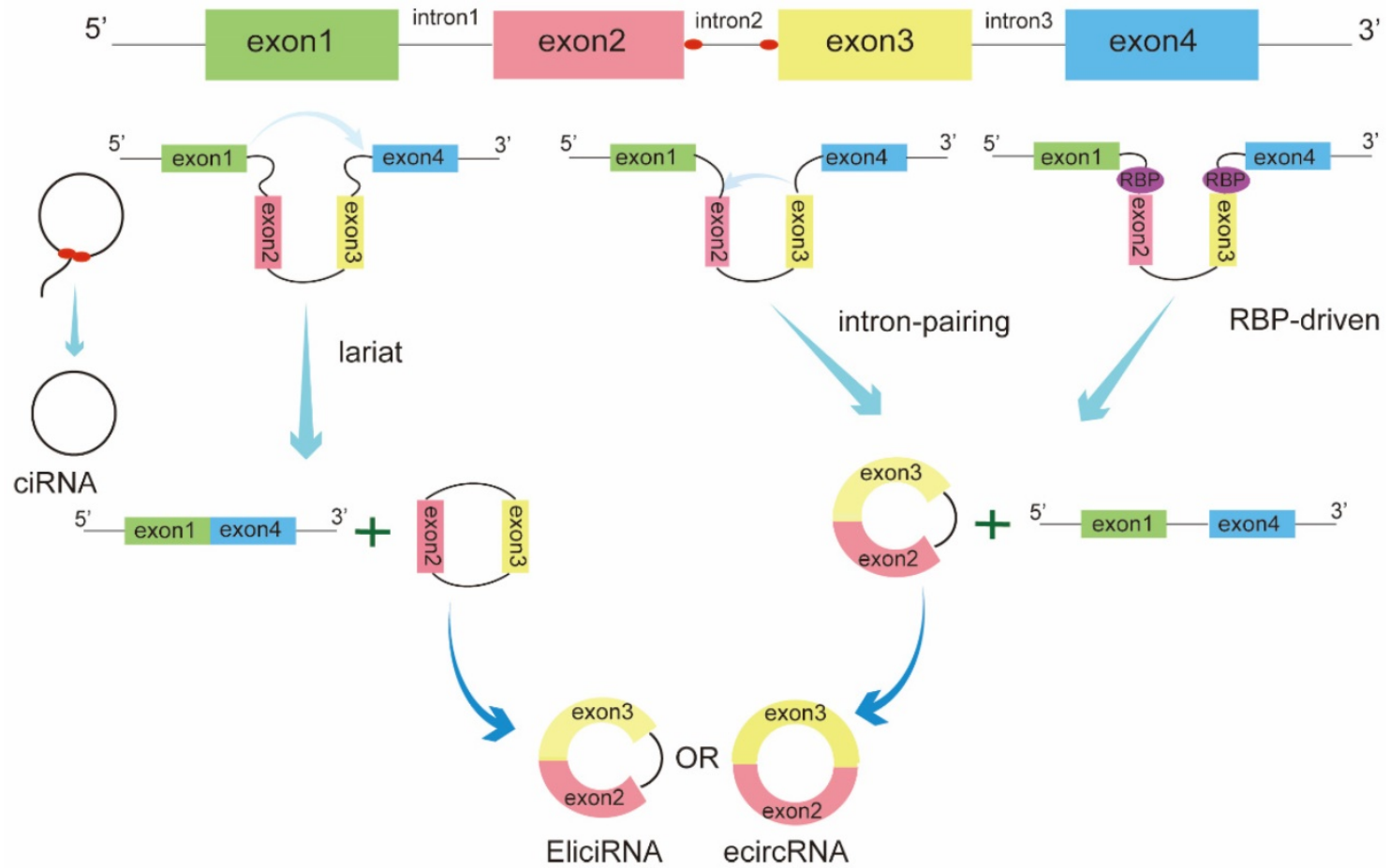

Figure 1. Biogenesis of circRNAs. The biogenesis of circRNAs is mainly regulated by three different mechanisms: lariat-driven circularization, intron pairing-driven circularization, and RNA-binding proteins (RBPs)-mediated circularization. 


\section{Functions of circRNA}

\section{1. miRNA sponge}

MicroRNAs (miRNAs) are important gene expression regulators, binding to specific sites of mRNA to prevent its translation or promote its degradation[17]. Increasing evidence has demonstrated that circRNAs can serve as competitive endogenous RNAs (ceRNAs) or miRNA sponges to inhibit miRNA function[18, 19]. In the pioneering study, a circRNA, named CDR1as (ciRS-7) was uncovered, which harbored $>70$ conserved miR-7 binding sites, and is a potent sponge of miR-7[20]. Moreover, Circ-ITCH is a circular RNA that could regulate the expression of $\mathrm{ITCH}$ by binding to multiple miRNAs[21, 22].

\subsection{Interaction with RBPs}

The circRNAs can bind to RBPs and function as RBP sponges. The ciR-7/CDRlas was reported to correlate with Argonaute (AGO) proteins in a miR-7-dependent manner[18]. The RBPs, which was found in flies and humans, can bind to the muscleblind (MBL) protein at multiple binding sites and promote its circularization to form circMBL[14]. In addition, RBPs are involved in almost all cellular processes, including proliferation, apoptosis, and differentiation[23].

\subsection{Regulation of gene transcription}

Circular RNAs are known to regulate gene transcription. Advanced studies have revealed that ciRNAs and EIciRNAs are primarily located in the nucleus and tend to function at the transcription level. The ciRNAs or EIciRNAs, like c-sirt7, are derived from lariats and can interact with the polymerase II complex, downregulating the expression of the corresponding genes ankyrin repeat domain 52 (ANKRD52) and sirtuin 7 (SIRT7)[24]. Thus, during the formation of circRNAs, it can modulate the expression of their parental genes, and this is one of the preconditions for the other functions of the circRNAs.

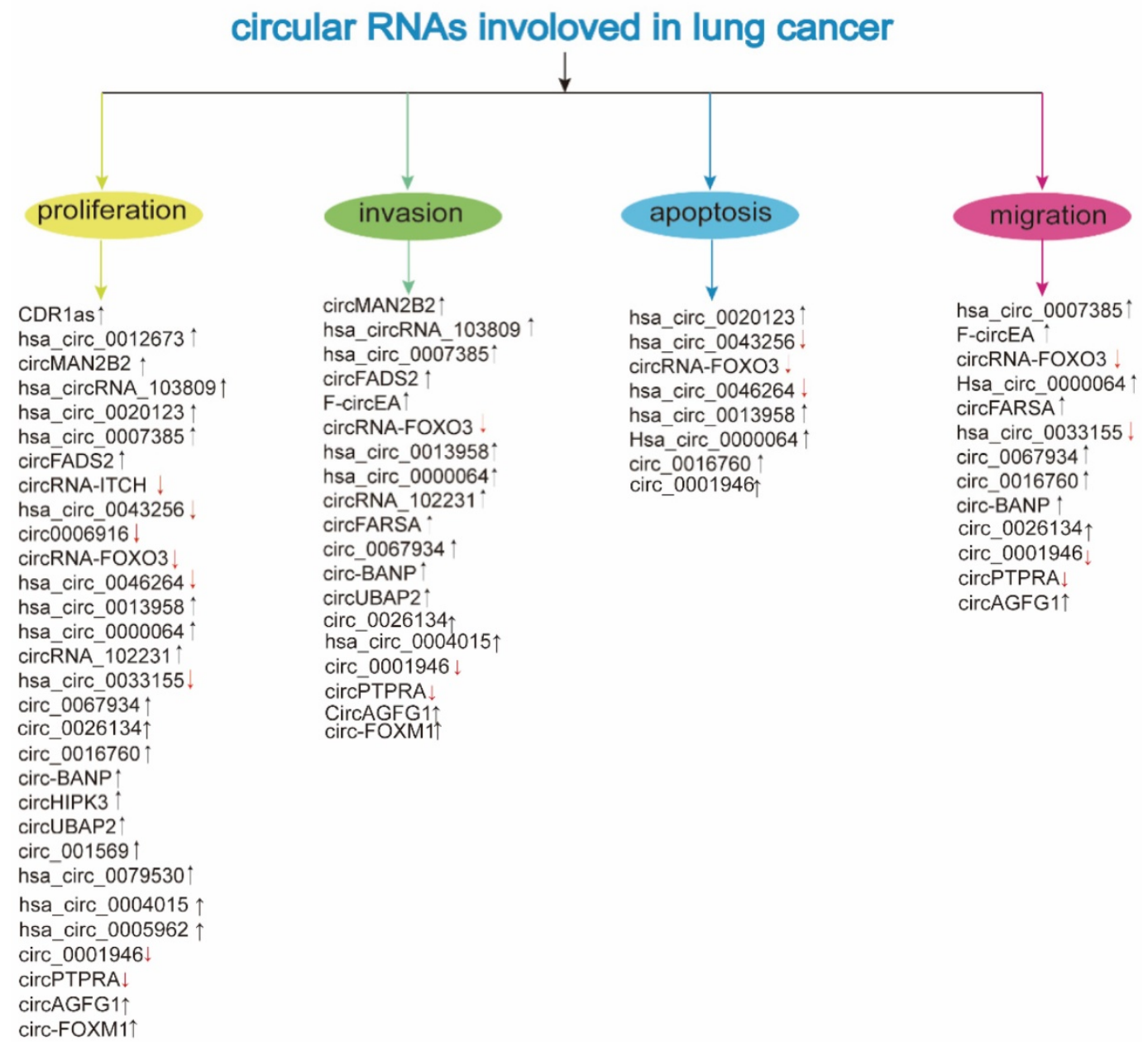

Figure 2. Involvement of circRNAs in lung cancer ( $\downarrow$ : downregulation; $\uparrow:$ upregulation; see the text for further details) 


\subsection{Translation to proteins}

In addition to the non-coding functions of circRNAs, scientists recently confirmed that circRNAs could also be translated to proteins. As early as 1995, scientists found that the internal ribosome entry site (IRES) of the encephalomyocarditis virus played an important role in the translation of an artificial circRNA, indicating that circRNAs might have the potential to encode proteins[25, 26]. Legnini et al. found that circ-ZNF609, which functions in myogenesis, could translate proteins in murine myoblasts when driven by IRES, providing the first proof that endogenous circRNAs have translational capabilities[27].

\section{Functional roles of circRNAs in LC}

The circRNAs are abnormally expressed and play an endogenous regulatory role in the carcinogenesis and development of LC. The expression and function of deregulated circRNAs are mentioned in Table 1 and Table 2, respectively.

Table 1. Upregulated circRNAs in lung cancer (LC)

\begin{tabular}{|c|c|c|c|c|c|}
\hline CircRNA & Expression Change & Function & Clinical relevance & Possible Mechanism & Reference \\
\hline CDR1as & $\mathrm{Up}$ & proliferation $(+)$ & $\begin{array}{l}\text { TNM } \\
\text { LNM } \\
\text { OS }\end{array}$ & miRNA sponge & [28] \\
\hline hsa_circ_0012673 & Up & proliferation $(+)$ & tumour size & miRNA sponge & [32] \\
\hline circMAN2B2 & Up & $\begin{array}{l}\text { proliferation }(+) \\
\text { invasion }(+)\end{array}$ & Unknown & circMAN2B2/miR-1275/FOXK1 signaling & [33] \\
\hline hsa_circRNA_103809 & Up & $\begin{array}{l}\text { proliferation }(+) \\
\text { invasion }(+)\end{array}$ & Unknown & hsa_circRNA_103809/miR-4302/ZNF121/MYC & [34] \\
\hline hsa_circ_0020123 & Up & $\begin{array}{l}\text { Proliferation }(+) \\
\text { apoptosis }(-)\end{array}$ & $\begin{array}{l}\text { TNM } \\
\text { LNM }\end{array}$ & hsa_circ_0020123-miR-144- ZEB1/EZH2 & [35] \\
\hline hsa_circ_0007385 & Up & $\begin{array}{l}\text { Proliferation }(+) \\
\text { migration }(+) \\
\text { invasion }(+)\end{array}$ & tumor growth & miRNA sponge & [36] \\
\hline circFADS2 & Up & $\begin{array}{l}\text { proliferation }(+) \\
\text { invasion }(+)\end{array}$ & $\begin{array}{l}\text { TNM } \\
\text { LNM } \\
\text { OS }\end{array}$ & miRNA sponge & [37] \\
\hline F-circEA & Up & $\begin{array}{l}\text { migration(+) } \\
\text { invasion }(+)\end{array}$ & Unknown & Not mentioned & {$[38,39]$} \\
\hline circ_0026134 & Up & $\begin{array}{l}\text { proliferation }(+) \\
\text { Migration }(+) \\
\text { invasion }(+)\end{array}$ & Unknown & miRNA sponge & [41] \\
\hline hsa_circ_0004015 & Up & $\begin{array}{l}\text { proliferation }(+) \\
\text { invasion }(+)\end{array}$ & OS & circ_0016760/miR-1183/PDPK1 & [42] \\
\hline circAGFG1 & Up & $\begin{array}{l}\text { proliferation }(+) \\
\text { Migration }(+) \\
\operatorname{EMT}(+)\end{array}$ & Unknown & Not mentioned & [43] \\
\hline Hsa_circ_0000064 & Up & $\begin{array}{l}\text { proliferation }(+) \\
\text { migration }(+) \\
\text { invasion }(+) \\
\text { apoptosis(-) }\end{array}$ & $\begin{array}{l}\text { T stage } \\
\text { TNM } \\
\text { LNM }\end{array}$ & Not mentioned & [51] \\
\hline circRNA_102231 & Up & $\begin{array}{l}\text { proliferation }(+) \\
\text { invasion }(+)\end{array}$ & $\begin{array}{l}\text { TNM } \\
\text { LNM } \\
\text { OS }\end{array}$ & Not mentioned & [52] \\
\hline hsa_circ_0005962 & Up & proliferation $(+)$ & Unknown & Not mentioned & [55] \\
\hline hsa_circ_0014130 & Up & Not mentioned & $\begin{array}{l}\text { TNM } \\
\text { LNM }\end{array}$ & miRNA sponge & [53] \\
\hline circFARSA & Up & $\begin{array}{l}\text { migration }(+) \\
\text { invasion }(+)\end{array}$ & Unknown & miRNA sponge & {$[57]$} \\
\hline circ_0067934 & Up & $\begin{array}{l}\text { proliferation }(+) \\
\text { migration }(+) \\
\text { invasion }(+) \\
\operatorname{EMT}(+)\end{array}$ & $\begin{array}{l}\text { TNM } \\
\text { LNM } \\
\text { OS }\end{array}$ & miRNA sponge & [58] \\
\hline circRNA_100876 & Up & Not mentioned & $\begin{array}{l}\text { LNM } \\
\text { tumor staging } \\
\text { OS }\end{array}$ & miRNA sponge & [59] \\
\hline circ_0016760 & $\mathrm{Up}$ & $\begin{array}{l}\text { proliferation }(+) \\
\text { migration }(+) \\
\text { apoptosis(-) }\end{array}$ & $\begin{array}{l}\text { TNM } \\
\text { LNM }\end{array}$ & circ_0016760/miR-1287/GAGE1 & [60] \\
\hline circ-BANP & Up & $\begin{array}{l}\text { proliferation }(+) \\
\text { invasion }(+) \\
\text { migration }(+)\end{array}$ & OS & circ-BANP-mediated miR-503/LARP1 & [62] \\
\hline hsa_circ_0000792 & Up & Not mentioned & TNM & miRNA sponge & [63] \\
\hline circHIPK3 & Up & $\begin{array}{l}\text { proliferation }(+) \\
\text { migration }(+) \\
\text { invasion }(+)\end{array}$ & Unknown & miRNA sponge & {$[64,65]$} \\
\hline
\end{tabular}




\begin{tabular}{|c|c|c|c|c|c|}
\hline CircRNA & Expression Change & Function & Clinical relevance & Possible Mechanism & Reference \\
\hline circUBAP2 & Up & $\begin{array}{l}\text { proliferation }(+) \\
\text { invasion }(+)\end{array}$ & Unknown & miRNA sponge & [66] \\
\hline circ_001569 & Up & proliferation $(+)$ & $\begin{array}{l}\text { LNM } \\
\text { TNM } \\
\text { OS }\end{array}$ & Wnt $/ \beta$-catenin & [67] \\
\hline hsa_circ_0079530 & Up & $\begin{array}{l}\text { Proliferation }(+) \\
\text { migration }(+) \\
\text { invasion }(+) \\
\text { EMT }(+)\end{array}$ & $\begin{array}{l}\text { LNM } \\
\text { tumor size }\end{array}$ & Not mentioned & {$[68]$} \\
\hline circ-FOXM1 & Up & $\begin{array}{l}\text { proliferation }(+) \\
\text { invasion }(+)\end{array}$ & $\begin{array}{l}\text { LNM } \\
\text { TNM }\end{array}$ & $\begin{array}{l}\text { circ-FOXM1/miR-1304-5p/PPDPF/MACC1 } \\
\text { signaling }\end{array}$ & \\
\hline
\end{tabular}

Table 2. Downregulated circRNAs in lung cancer (LC)

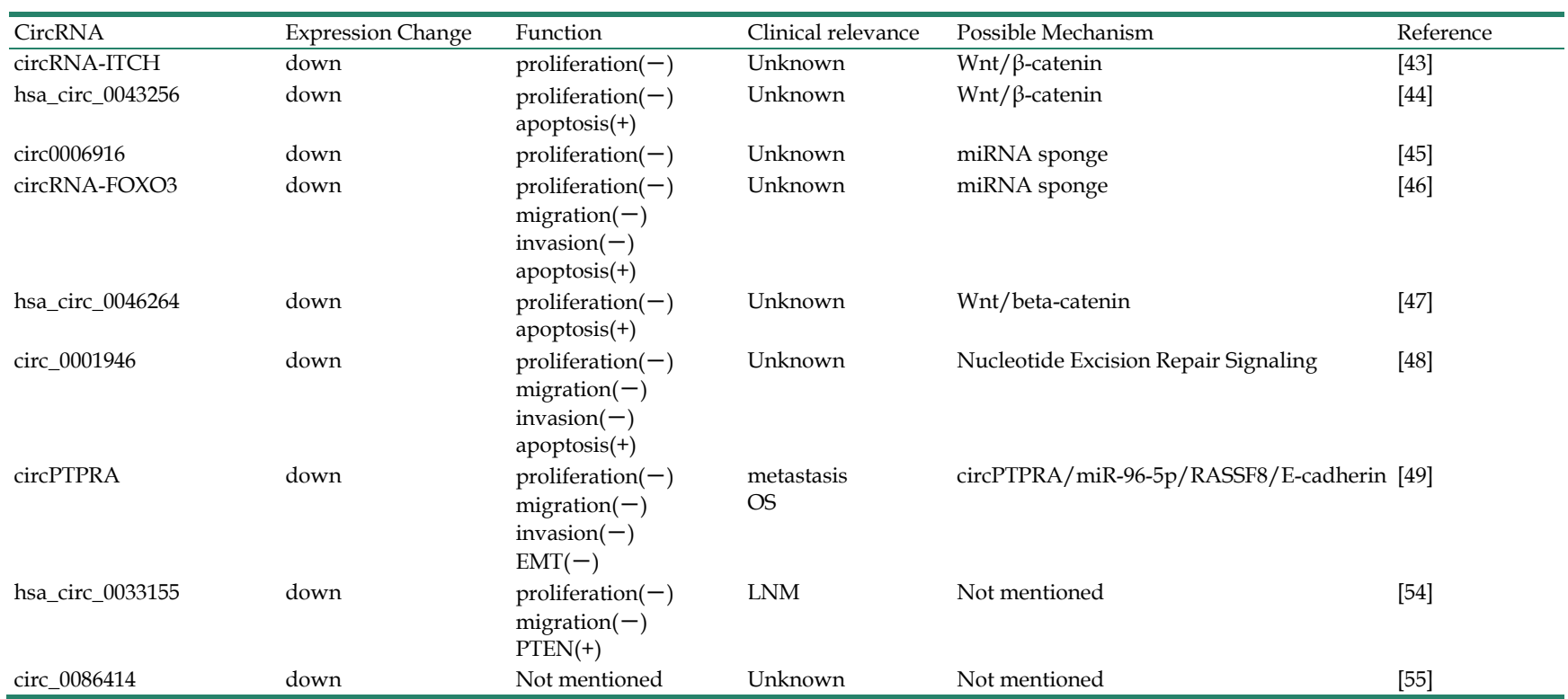

\subsection{Oncogenes}

The extensively investigated circRNA, CDR1as, has been reported to be deregulated in a variety of cancers, including LC, and the first evidence of the function of circRNA as a microRNA sponge was the interaction between circular RNA ciRS-7 (CDR1as) and miRNA-7 (miR-7). The study showed a high expression of CDR1as in NSCLC tissues, which was strikingly associated with the high stage of tumor Node Metastasis(TNM); lymph node metastasis (LNM), and shorter overall survival (OS)[28]. Further, it was reported that CDR1as levels in tumor samples were negatively related to the expression of miR-7, which showed that the CDR1as might inhibit miR-7 in NSCLC. Overexpression of miR-7 restrained the gene expression of epidermal growth factor receptor (EGFR), CCNE1, and PIK3CD, which were restored by the overexpression of CDR1as. Moreover, CDR1as promoted cell growth via the miR-7/ EGFR/ CCNE1/PIK3CD signals in NSCLC. These findings demonstrated that CDR1as functioned as an oncogene to inhibit the anti-tumor effects of tumor suppressor miR-7 by the up-regulation of proliferation index
Ki-67, EGFR, CCNE1, and PIK3CD levels. The ErbB3 plays a major role in carcinogenesis and cancer progression. It was dramatically upregulated in LC, breast cancer, ovarian cancer, colorectal cancer, and gastric cancer[29]. High expression of ErbB3 correlated with tumor proliferation and migration in LC[30] and miR-22 inhibited the progression of LUAD through post-transcriptional regulation of ErbB3[31]. Wang et al. indicated that hsa_circ_0012673 acted as a miR-22 sponge, and thus, regulated ErbB3 expression[32]. The hsa_circ_0012673 was found to be up-regulated in LUAD tissues versus adjacent tissue, and the expression level of hsa_circ_100338 was tightly associated with the tumor size. Furthermore, hsa_circ_0012673 could promote cell proliferation by sponging miR-22, which targeted ErbB3 in LUAD. The circMAN2B2 was reported to be highly expressed in the LC tissues[33]. The knockdown of circMAN2B2 repressed the proliferation and invasion of LC cells. Furthermore, the downregulation of circMAN2B2 enhanced the expression of miR-1275 in LC and inhibited the levels of its target, FOXK1. Taken together, circMAN2B2 functioned as an oncogene in LC through promoting FOXK1 expression by 
sponging miR-1275. The hsa_circRNA_103809 was overexpressed in LC tissues[34], and its knockdown significantly inhibited the proliferation and invasion of LC cells and delayed tumor growth in vivo. The hsa_circRNA_103809 functioned as a sponge of miR-4302 by promoting the expression of ZNF121, thus enhancing the MYC protein levels in the LC cells. The MYC is a classical oncoprotein in various cancers, including LC, and an in-depth investigation demonstrated the significance of the hsa_circRNA_103809/miR-4302/ZNF121/MYC regulatory in LC progression. In summary, these findings illustrated that hsa_circRNA_103809 performed an oncogene function in LC and might be a potential therapeutic target. $\mathrm{Qu}$ et al. found a notable increase in the expression of hsa_circ_0020123 in cancer tissues compared to the normal lung tissues[35]. The upregulation of hsa_circ_0020123 was tightly associated with a poor differentiation degree, LNM, high TNM stage, and dismal prognosis in NSCLC patients. Overexpression of hsa_circ_0020123 significantly promoted tumor growth and metastasis in NSCLC cells, indicating that hsa_circ_0020123 could promote NSCLC progression by sponging miR-144. In addition, hsa_circ_0020123 could upregulate ZEB1 and EZH2 through competitively binding with miR-144. Finally, silencing hsa_circ_0020123 by siRNA delivery was shown to suppress NSCLC growth and metastasis in vivo. These findings suggested that hsa_circ_0020123-miR-144ZEB1/EZH2 axis is critical for NSCLC progression, which indicates that hsa_circ_0020123 is a promising therapeutic for NSCLC treatment. Jiang et al. screened circRNA expression profiles and identified a total of 957 abnormally expressed circRNAs in NSCLC tissues compared to the normal tissues[36]. Studies have shown that hsa_circ_0007385 was significantly up-regulated in NSCLC tissue, and its knockdown suppressed the proliferation, migration, and invasion of NSCLC cells in vitro. Specifically, the downregulation of hsa_circ_0007385 enhanced the expression of miR-181. Thus, upregulated circRBM23 functioned as an oncogene in HCC through the downregulation of the tumor suppressor miR-181. Zhao et al. highlighted that circFADS2 expression was significantly upregulated in LC tissues and correlated with the advanced TNM stage, LNM, poor differentiation, and poor OS of NSCLC patients[37]. Further analysis indicated that circFADS2 knockdown inhibited the proliferation and invasion ability of the NSCLC cells. The circFADS2 could act as an oncogenic circRNA in LC progression by sponging miR-498. Taken together, these results suggested that circFADS2 could be a promising therapeutic target for LC treatment. The oncogenic fusion gene,
Echinoderm Microtubule-associated protein-Like 4-Anaplastic Lymphoma Kinase (EML4-ALK), plays a vital role in the tumorigenesis of NSCLC. Tan et al. tested the EML4-ALK-positive cell line H2228 and found that the fusion-circRNA from EML4-ALK fusion gene (F-circEA) could promote cell migration and invasion[38]. Moreover, they indicated that another circRNA, F-circEA-2a, produced from EML4-ALK-v3b, is located in the cytoplasm and promoted cell migration and invasion[39]. The F-circEA-2a exists in the tumor, but not in the plasma of NSCLC patients with EML4-ALK fusion gene, further indicating that F-circEA has a significant diagnostic value for the diagnosis of EML4-ALKpositive NSCLC. Fusion-circRNA (F-circRNA) has been reported to have tumor-promoting properties in vivo, functioning as a diagnostic marker for the EML4/ALK1 gene fusion mutation in LC[40]. Multiple tumors are correlated with recurrent chromosomal translocations and the transcription of genes affected by chromosomal translocations could allow the formation of new circRNAs, namely F-circRNAs. Moreover, LC-associated EML4/ALK1 translocation originated from F-circRNAs, which are functionally relevant (tumor promoting), and represented a novel entry point for LC diagnosis. The circ_0026134 was highly expressed in lung tumors versus the normal adjacent tissue[41]. The knockdown of circ_0026134 significantly inhibited the proliferation and metastatic properties of NSCLC cells and induced cell apoptosis. Mechanistically, circ_0026134 acted as a sponge for miR-1256 and miR-1287, and thus, facilitates cell proliferation and invasion. Consequently, the overexpression of circ_0026134 acts as an oncogene in NSCLC cells. Zhou et al.[42] showed that hsa_circ_0004015 was upregulated in NSCLC tissues and enhanced cell viability, proliferation, and invasion, whereas, its silencing exhibited opposite effects. The upregulation of hsa_circ_0004015 was tightly associated with the poor OS in NSCLC patients. Furthermore, hsa_circ_100338 served as a sponge for miR-1183 and induced the expression of the 3-phosphoinositidedependent protein kinase 1 (PDPK) and stimulated AKT pathway. The circRNA, circAGFG1, was reported to have an oncogenic role in LC progression[43]. This result was obtained by real-time PCR analysis of 20 pairs of NSCLC tissues and their adjacent tissues. Subsequent experimental results proved that the silencing of circAGFG1 inhibited the proliferation and migration of A549 and H1299 cells. Mechanistic analyses demonstrated that circAGFG1 could act as a sponge for miR-203 to repress its effect on its target, ZNF281. 


\subsection{Tumor Suppressors}

The circRNA-ITCH and hsa_circ_0043256 functioned as tumor suppressors in LC by up-regulating ITCH expression and inhibiting the activation of $\mathrm{Wnt} / \beta$-catenin pathway; the expression of cir-ITCH was found to be decreased in LC[43]. As a circRNA, cir-ITCH could regulate the expression of ITCH by sponging miR-7 and miR-214, and consequently, inactivating LC cell proliferation. Further studies show that circ-ITCH inactivated Wnt/ $\beta$ catenin pathway through transcriptionally regulated T-cell factor, $\beta$-catenin, c-Myc, and cyclinD1. The Wnt/ $\beta$-catenin pathway was reported to play a critical role in tumorigenesis, and aberrant activation of the Wnt/ $\beta$-catenin pathway was closely associated with NSCLC progression. Cinnamaldehyde (CA) has been reported to trigger NSCLC apoptosis through the inhibition of Wnt/ $\beta$-catenin pathway in three kinds of NSCLC cells[44]. The expression of hsa_circ_0043256 was increased in NSCLC in response to CA treatment in vitro and in vivo. Overexpression of hsa_circ_0043256 could inhibit cell proliferation and induce apoptosis, whereas hsa_circ_0043256 knockdown resulted in the opposite effect. Moreover, hsa_circ_0043256 could function as a miR-1252 sponge, affecting the important negative regulator of the Wnt signaling pathway. These results suggested an important role of the hsa_circ_0043256/miR-1252/ITCH axis in the anti-tumor activity mediated by CA. The circ0006916 was down-regulated in LC cells and tissues. Overexpression of circ0006916 could affect the cell cycle distribution and inhibit cell proliferation[45]. Moreover, circ0006916 could act as a microRNA sponge in LC progression and exert regulatory functions by binding to miR-522-3p. These results indicate that circ0006916 may serve as a tumor suppressor in LC. The circRNA-FOXO3 may serve as a tumor suppressor in LC through sequestering miR-155 and enhancing FOXO3 expression[46]. Zhang et al. found that the expression of circRNA-FOXO3 was lower in NSCLC tissues and cell lines, and its upregulation significantly suppressed cell proliferation, migration, and invasion and promoted apoptosis. Mechanistically, circRNAFOXO3 increases the expression of $\mathrm{FOXO} 3$ by sequestering miR-155, and thus, functions as a tumor-suppressor gene. Yang et al. investigated the expression and function of hsa_circ_0046264 in LC and reported its downregulation[47]. The overexpression of hsa_circ_0046264 significantly decreased the expression of miR-1245 and enhanced the levels of its target gene, BRCA2. Moreover, it has been found that hsa_circ_0046264 suppressed LC development and tumor growth in vivo. Liu et al. found that the expression of hsa_circ_0001946 was reduced in human lung cancer tissues and cell lines[48]. The knockdown of hsa_circ_0001946 promoted NSCLC cell viability, proliferation, migration, and invasion, as well as inhibition of cell apoptosis. More importantly, hsa_circ_0001946 downregulation activated the NER signaling pathway, which in turn reduced cisplatin sensitivity and increased DNA repair ability. These results confirmed that hsa_circ_0001946 not only performs an inhibitory function in NSCLC tumorigenesis but also might be a novel biomarker for predicting survival probability and the chemosensitivity of cisplatin in lung cancer patients. The expression of circPTPRA was dramatically downregulated in NSCLC tissues compared to the healthy lung tissues[49]. The downregulation of circPTPRA in NSCLC was associated with the clinicopathological features of NSCLC patients, including metastasis and shorter survival. In terms of mechanism, circPTPRA mediates its EMT-suppressive effects by sequestering miR-96-5p, which upregulates the downstream tumor suppressor Ras association domain-containing protein 8(RASSF8). These results advocated the circPTPRA/miR-96-5p/ RASSF8/Ecadherin axis as a new perspective on the role of circRNAs in NSCLC development.

\subsection{Diagnostic value}

Zhao et al. investigated the expression profile of circRNAs in early-stage lung adenocarcinoma tissues versus the normal tissues[50]. They identified a total of 357 circRNAs that were dysregulated in the early-stage lung adenocarcinoma, including 204 upregulated circRNAs and 152 downregulated circRNAs. Among these circRNAs, hsa_circRNA_404833 and hsa_circRNA_406483 displayed significant differences in their expression between LC tissues and normal tissues, which demonstrated that both might be exceptional potential candidates as the early diagnostic biomarker for LC. They also indicated that hsa_circRNA_404833 is a potential sponge of miR-149-5p, which inhibits cell motility and is involved in the acquired gefitinib resistance in LC. The hsa_circ_0000064 might act as a new promising biomarker and therapeutic target of LC[51] as it was remarkably up-regulated in LC tissues and cell lines, which were related to the $\mathrm{T}$ stage, lymphatic metastasis, and TNM stage. The hsa_circ_0000064 knockdown suppressed cell proliferation, promoted cell apoptosis, and blocked cell cycle progression in A549 and H1229 cells. Western blot data further confirmed that the knockdown of hsa_circ_0000064 increased the level of the apoptosis-related $\mathrm{Bcl}-2$ protein, while the 
expression of the cancer-related proteins involved in cell cycle, cell apoptosis, migration, and invasion was considerably restrained in the A549 and H1229 cells. This result indicated that hsa_circ_0000064 represented a promising biomarker for LC diagnosis. The circRNA_102231 might act as a potential therapeutic target for LC[52]. Zong et al. found that circRNA_102231 expression increases in LAC tissues and its high expression was closely implicated in the advanced TNM stage, LNM, and poor OS of LC patients. Function assays further confirmed that circRNA_102231 inhibition reduced the LC cells proliferation and invasive ability. Zhang et al. revealed the circRNA expression profiles in NSCLC tissues and their adjacent lung tissue and found that hsa_circ_0014130 (circPIP5K1A) expression was higher and its expression levels were associated with the TNM stage and lymphatic metastasis[53]. Gene oncology analysis and pathway analysis showed that hsa_circ_0014130 had a strong relationship with NSCLC development. More importantly, hsa_circ_0014130 might interact with five miRNAs and their corresponding mRNAs. Taken together, these studies revealed that hsa_circ_0014130 has a diagnosis value in distinguishing NSCLC from non-cancerous tissues. $\mathrm{Gu}$ et al. indicated that hsa_circ_0033155 was significantly downregulated in the NSCLC tissue and its dysregulation can be associated with lymphatic metastasis[54]. Furthermore, overexpression of hsa_circ_0033155 in NSCLC cells decreased cell proliferation, inhibits colony formation, and migration. Accordingly, hsa_circ_0033155 possesses a promising potential as a biomarker and a therapeutic target for NSCLC.

The circRNAs are insensitive to ribonucleases due to their stable circular structures, and thus, make them more stable and particularly attractive as liquid biopsy biomarkers. Recently, a global circRNA expression was detected using bioinformatics analysis[55]. The hsa_circ_0005962 was shown to be significantly upregulated in LUAD plasma and cells, whereas hsa_circ_0086414 was downregulated. The ROC curve analysis suggested that a signature comprising the two circRNAs had good diagnostic potential for LUAD. Moreover, high plasma hsa_circ_0086414 expression level was related to EGFR mutations. The hsa_circ_0005962 expression level was decreased after surgery in patients with LUAD, but hsa_circ_0086414 was not significantly different after surgery. Zhu et al. used a microarray technique for the detection of tumor-specific circRNA candidates in LAC tissue, and they found 39 upregulated and 20 downregulated circRNAs[56]. Among them, hsa_circ_0013958 was shown to be upregulated in all the LAC tissues, cells, and plasma.
Mechanistically, they found that hsa_circ_0013958 acted as a sponge for miR-134, and thus, up-regulated the oncogene CCND1, which plays a critical role in the development of NSCLC. Further analysis confirmed that hsa_circ_0013958 levels were correlated with TNM stage and lymphatic metastasis. These findings suggested that the expression level of hsa_circ_0013958 in stage I/II LAC patients was significantly up-regulated compared to the healthy controls, indicating that hsa_circ_0013958 could serve as a non-invasive biomarker, having high sensitivity and specificity, for the early detection and screening of LAC. A total of 185 differentially expressed circRNAs were identified in NSCLC tissues, including 120 upregulated circRNAs and 65 downregulated circRNAs[57]. The circFARSA was shown to be higher in cancerous tissues and was more abundant in patients' plasma than the controls. Functional analysis demonstrated that circFARSA overexpression promoted NSCLC cell migration and invasion. It might contribute to the development of LC by sponging several miRNAs, including miR-330- $5 p$ and miR-326. These findings suggested that circFARSA was a noninvasive biomarker for NSCLC.

\subsection{Prognostic value}

Recently, circ_0067934 was reported to be overexpressed in NSCLC tissues and cell lines, and its elevated expression was significantly associated with the advanced TNM stage, lymph node status, distant metastasis[58]. Further study revealed that the circ_0067934 expression, which was related to a poorer OS, was used as a prognostic marker for invasive NSCLC. Moreover, circ_0067934 could regulate E2F7 as a miR-545 and miR-589 sponge. The expression level of circRNA_100876 was elevated in NSCLC tissues in the clinical samples[59]. High expression of circRNA_100876 was significantly correlated with LNM and advanced tumor staging in NSCLC. In addition, increased circRNA_100876 expression correlates with a short OS time in NSCLC patients. In conclusion, these findings demonstrated that circRNA_100876 is closely related to the carcinogenesis of NSCLC, and it could be used as a potential prognostic biomarker and therapeutic target for NSCLC. The circ_0016760 was highly expressed in NSCLC, and the upregulation of circ_0016760 was closely connected with advanced TNM stage, LNM, and adverse prognosis in NSCLC patients[60]. Moreover, the survival curve demonstrated that high levels of circ_0016760 were associated with shorter OS in NSCLC patients. Therefore, circ_0016760 might be a prognostic factor for NSCLC patients. Mechanistically, circ_0016760 acts as a sponge for miR-1287 and regulates GAGE1 expression. In 
summary, circ_0016760 might be involved in the tumorigenesis of NSCLC by circ_0016760/miR-1287/ GAGE1 signaling. $\mathrm{Xu}$ et al. investigated the expression profile of circular RNA in LUSC. Among the differentially expressed circRNAs, hsa_circRNA_000122 was identified to be dramatically downregulated compared to the matched adjacent normal tissues, while hsa_circRNA_103827 was highly expressed[61]. The results indicated that the OS time of LSCC patients with high hsa_circRNA_103827 expression and low hsa_circRNA_000122 was significantly shorter. Consequently, has_circRNA__103827 may be a promising biomarker for LC prognosis. Han et al. revealed that the circ-BANP expression, which was related to lower survival rate, increased in LC tissues[62]. Functionally, silencing circ-BANP inhibited cell proliferation and migration and induced invasion in vitro and delayed the progression of LC in vivo. Importantly, circ-BANP acts as a miR-503 sponge to upregulate the expression of LARP1. Collectively, the circ-BANP-mediated miR-503/LARP1 signaling performed a modulatory role in LC tumorigenesis, providing a promising prognostic indicator in $\mathrm{LC}$. $\mathrm{Li}$ et al. found that hsa_circ_0000792 was significantly upregulated in LUAD compared with the matched noncancerous tissues[63]. The hsa_circ_0000792 was involved in signal transduction and cell communication in LUAD development. Further analysis showed that hsa_circ_0000729 expression had a negative correlation with miR-375. These findings supported that hsa_circ_0000792 holds great potential for LUAD diagnosis and prognosis. The circHIPK3 was found to be commonly expressed in six different NSCLC cell lines, with the highest expression in NCI-H2170 and the lowest in NCI-H1299[64]. The upregulation of circHIPK3 was able to stimulate cell proliferation in NCI-H2170. In contrast, interference with circHIPK3 expression was shown to inhibit NCI-H2170 cell proliferation. Moreover, circHIPK3 could sponge miR-379, which increased the levels of its target gene, IGF1. Thus, circHIPK3 performed a proliferation-promoting function in LC and could be a novel therapeutic target for NSCLC. Chen et al.[65] also confirmed that the knockdown of circHIPK3 significantly suppressed NSCLC cell proliferation, migration, and invasion and induced macroautophagy/autophagy. The circHIPK3 silencing was shown to inhibit cell growth and might be partially associated with the induction of autophagy in a subset of LC cell lines. Further study indicated that circHIPK3 abrogation induced autophagy via STAT3-PRKAA and was STK11-dependent. It functions as an oncogene and autophagy regulator, and might be a potential prognostic indicator and therapeutic target in LC. Loss-of-function studies indicated that circUBAP2 knockdown suppressed the proliferation and invasion of lung adenocarcinoma[66]. In vitro study showed that circUBAP2 silencing inhibited the expression of CDK6, cyclin D1, c-IAP1, Bcl-2, SuUBRrvivin, FAK, Rac1, and MMP2, while the expression of p27 and Bax was significantly increased. Moreover, circUBAP2 knockdown can suppress the activities of Rac1 and FAK. Consequently, circUBAP2 plays an important role in the proliferation and invasion of human LC and is a promising target for the treatment of NSCLC. Ding et al. compared the expression of circ_001569 between paired NSCLC and adjacent normal tissues[67]. The result indicated that the expression of circ_001569 was increased in NSCLC tissues. The upregulation of circ_001569 in NSCLC was strikingly associated with tumor differentiation, LNM, and TNM classification. Functional assay results indicated that circ_001569 knockdown suppressed the proliferation of NSCLC in vitro. In addition, circ_001569 knockdown could inhibit the Wnt/ $\beta$-catenin signaling pathway in NSCLC cells. Therefore, the high expression of circ_001569 might present a risk factor for predicting the prognosis of patients with NSCLC. $\mathrm{Li}$ et al. proposed that hsa_circ_0079530 was upregulated in LC tissues and tightly associated with tumor size and LNM[68]. The hsa_circ_0075930 knockdown suppressed the proliferation and also induced the migratory and invasive capabilities of NSCLC cells by reversing epithelial-mesenchymal transition (EMT), which is associated with a more invasive phenotype in tumors. This data suggests that hsa_circ_0079530 performed an oncogenic role in NSCLC and may serve as a prognosis predictor and therapeutic target for NSCLC. Liu et al.[69] found that the circ-FOXM1 expression, which was closely associated with lymph node invasion, higher TNM stage, and unfavorable prognosis, was upregulated in the lung cancer tissues. The circ-FOXM1 promoted the proliferation and invasion of NSCLC cells by upregulating the levels of PPDPF and MACC1 through sponging miR-1304-5p. This finding indicated that circ-FOXM1/ miR-1304-5p/PPDPF/MACC1 signaling is essential for the development and progression of NSCLC.

\section{Conclusions}

Lung cancer is the root cause of cancer-associated deaths globally. Considering the high morbidity and mortality of LC, many trials are in progress, looking for more advanced therapeutic approaches. Targeted therapy is now one of the important hot spots of cancer treatment research; circular RNA is a new frontier in cancer research. It 
has been indicated that altered circRNA expression can affect the tumorigenesis and progression of LC, and these circRNAs hold important clinical relevance due to their advantages in LC diagnosis, therapy, and prognosis. Circular RNAs possess multiple advantages, including relatively long and stable structures, suggesting that circRNAs are ideal diagnostic biomarkers and promising therapeutic targets for LC, which could provide a novel avenue for us to develop a panel of specific circRNAs for different types of LCs that can be safely and successfully integrated into clinical practice. Recent studies have found that circRNAs function as miRNA sponges, regulate transcription, or affect gene/protein expression involved in the cell cycle, proliferation, invasion, and metastasis. However, compared with the other ncRNAs, the study of circRNAs in LC is still in the early stage, and only a small number of functional circRNAs have been discovered and characterized. Circular RNAs have a variety of important biological functions, but the existing research is mostly devoted to the miRNA sponge function of the circRNAs, which makes the related research not comprehensive or deep enough; other functions of circRNA require further study. The functional mechanism of the circular RNAs in LC is not limited to miRNA sponges; several studies have unveiled the other functions of circRNAs. The complex regulatory network of the competing endogenous RNA (ceRNA) needs to be enriched by incorporating more factors. The improvement in the second-generation sequencing technology and the reduction in cost, along with the establishment of an unbiased bioinformatic method will lead to the identification of a large number of novel circRNAs, whose potential mechanisms would be studies, which will accelerate the clinical application of circRNAs in LC diagnosis and therapy.

\section{Abbreviations}

LC: Lung cancer; circRNAs: circular RNAs; SCLC: small cell lung cancer; NSCLC: non-small cell lung cancer; LCLC: large cell lung carcinoma; LUSC: squamous cell carcinoma; LUAD: lung adenocarcinoma; ecircRNAs: exonic circRNAs; ciRNAs: circular intron RNAs; EIciRNAs: exon-intron circRNAs; ceRNAs: competitive endogenous RNAs; RBP: RNA-binding proteins; MBL: muscleblind; AGO: Argonaute; ANKRD52: ankyrin repeat domain 52; SIRT7: sirtuin 7; IRES: internal ribosome entry site; miR-7: miRNA-7; TNM: Tumor Node Metastasis; LNM: lymph node metastasis; OS: overall survival; CA: Cinnamaldehyde; EGFR: epidermal growth factor receptor; EML4-ALK: Echinoderm Microtubule-associated protein-Like 4-Anaplastic
Lymphoma Kinase; F-circEA: Fusion-circRNA from EML4-ALK; F-circRNA: Fusion-circRNA; PDPK: 3-phosphoinositide-dependent protein kinase 1; RASSF8: Ras association domain-containing protein 8; EMT: epithelial-mesenchymal transition; PPDPF: pancreatic progenitor cell differentiation and proliferation factor; MACC1: metastasis-associated in colon cancer 1 .

\section{Acknowledgements}

This study was funded by the Municipal Human Resources Development Program for Outstanding Young Talents in Medical and Health Sciences in Shanghai (Grant:2018YQ15, to Lifang Ma), supported by the Natural Science Foundation of China (Grant:81774291), and sponsored by Shanghai Sailing Program (Grant:18YF1421800, to Lifang Ma), "Chen Guang" project supported by the Shanghai Municipal Education Commission and Shanghai Education Development Foundation (Grant:17CG43, to Lifang $\mathrm{Ma})$, Innovation project of Shanghai University of Traditional Chinese Medicine (Grant JXDXSCXJH18, to $\mathrm{Xin} \mathrm{Xu}$ ).

\section{Competing Interests}

The authors have declared that no competing interest exists.

\section{References}

1. Bray F, Ferlay J, Soerjomataram I, Siegel RL, Torre LA, Jemal A. Global cancer statistics 2018: GLOBOCAN estimates of incidence and mortality worldwide for 36 cancers in 185 countries. CA Cancer J Clin. 2018; 68: 394-424.

2. Memczak S, Jens M, Elefsinioti A, Torti F, Krueger J, Rybak A, et al. Circular RNAs are a large class of animal RNAs with regulatory potency. Nature. 2013; 495: 333-8.

3. Faivre C, El Cheikh R, Barbolosi D, Barlesi F. Mathematical optimisation of the cisplatin plus etoposide combination for managing extensive-stage small-cell lung cancer patients. Br J Cancer. 2017; 116: 344-8.

4. Molina JR, Yang P, Cassivi SD, Schild SE, Adjei AA. Non-Small Cell Lung Cancer: Epidemiology, Risk Factors, Treatment, and Survivorship. Mayo Clinic Proceedings. 2008; 83: 584-94.

5. Ettinger DS,Wood DE,Aisner FL,Akerley W, Bauman J,Chirieac LR,et al. Non-Small Cell Lung Cancer, Version 5.2017, NCCN Clinical Practice Guidelines in Oncology. JNCCN. 2017; 15: 504-35.

6. Salzman J, Gawad C, Wang PL, Lacayo N, Brown PO. Circular RNAs are the predominant transcript isoform from hundreds of human genes in diverse cell types. PLoS One. 2012; 7: e30733.

7. JU G, V A, H G, DP B. Expanded identification and characterization of mammalian circular RNAs. Genome biology. 2014; 15: 409.

8. Suzuki H, Tsukahara T. A view of pre-mRNA splicing from RNase R resistant RNAs. Int J Mol Sci. 2014; 15: 9331-42.

9. Sanger HL, Klotz G, Riesner D, Gross HJ, Kleinschmidt AK. Viroids are single-stranded covalently closed circular RNA molecules existing as highly base-paired rod-like structures. Biochemistry. 1976; 73: 3852-6.

10. Meng X, Li X, Zhang P, Wang J, Zhou Y, Chen M. Circular RNA: an emerging key player in RNA world. Brief Bioinform. 2017; 18: 547-57.

11. Bolha L, Ravnik-Glavac M, Glavac D. Circular RNAs: Biogenesis, Function, and a Role as Possible Cancer Biomarkers. Int J Genomics. 2017; 2017: 6218353.

12. Jeck WR, Sorrentino JA, Wang K, Slevin MK, Burd CE, Liu J, et al. Circular RNAs are abundant, conserved, and associated with ALU repeats. Rna. 2012; 19: 141-57.

13. Conn SJ, Pillman KA, Toubia J, Conn VM, Salmanidis M, Phillips CA, et al. The RNA binding protein quaking regulates formation of circRNAs. Cell. 2015; 160: 1125-34. 
14. Ashwal-Fluss R, Meyer M, Pamudurti NR, Ivanov A, Bartok O, Hanan $\mathrm{M}$, et al. circRNA biogenesis competes with pre-mRNA splicing. Mol Cell. 2014; 56: 55-66.

15. Zhang XO, Wang HB, Zhang Y, Lu X, Chen LL, Yang L. Complementary sequence-mediated exon circularization. Cell. 2014; 159: 134-47.

16. Zhang $\mathrm{Y}$, Zhang XO, Chen $\mathrm{T}$, Xiang JF, Yin QF, Xing YH, et al. Circular intronic long noncoding RNAs. Mol Cell. 2013; 51: 792-806.

17. Beermann J, Piccoli MT, Viereck J, Thum T. Non-coding RNAs in Development and Disease: Background, Mechanisms, and Therapeutic Approaches. Physiol Rev. 2016; 96: 1297-325.

18. Hansen TB, Jensen TI, Clausen BH, Bramsen JB, Finsen B, Damgaard CK, et al. Natural RNA circles function as efficient microRNA sponges. Nature. 2013; 495: 384-8.

19. Sumazin P, Yang X, Chiu HS, Chung WJ, Iyer A, Llobet-Navas D, et al. An extensive microRNA-mediated network of RNA-RNA interactions regulates established oncogenic pathways in glioblastoma. Cell. 2011; 147: $370-81$

20. Memczak S, Jens M, Elefsinioti A, Torti F, Krueger J, Rybak A, et al. Circular RNAs are a large class of animal RNAs with regulatory potency. Nature. 2013; 495: 333.

21. Zheng Q, Bao C, Guo W, Li S, Chen J, Chen B, et al. Circular RNA profiling reveals an abundant circHIPK3 that regulates cell growth by sponging multiple miRNAs. Nat Commun. 2016; 7: 11215.

22. Wan L, Zhang L, Fan K, Cheng ZX, Sun QC, Wang JJ. Circular RNA-ITCH Suppresses Lung Cancer Proliferation via Inhibiting the Wnt/beta-Catenin Pathway. Biomed Res Int. 2016; 2016: 1579490.

23. Liu J, Liu T, Wang X, He A. Circles reshaping the RNA world: from waste to treasure. Mol Cancer. 2017; 16: 58.

24. Rong D, Tang W, Sun H, Li ZX, Liu SH, Dong CX, et al. An emerging function of circRNA-miRNAs-mRNA axis in human diseases. Oncotarget. 2017; 8: 73271-81.

25. Wang Y, Wang Z. Efficient backsplicing produces translatable circular mRNAs. RNA. 2015; 21: 172-9.

26. Chen CY, Sarnow P. Initiation of protein synthesis by the eukaryotic translational apparatus on circular RNAs. Science (New York, NY). 1995; 268: 415-7.

27. Legnini I, Di Timoteo G, Rossi F, Morlando M, Briganti F, Sthandier O, et al. Circ-ZNF609 Is a Circular RNA that Can Be Translated and Functions in Myogenesis. Mol Cell. 2017; 66: 22-37 e9.

28. Zhang X, Yang D, Wei Y. Overexpressed CDR1as functions as an oncogene to promote the tumor progression via miR-7 in non-small-cell lung cancer. Onco Targets Ther. 2018; 11:3979-87.

29. Sithanandam G, Anderson LM. The ERBB3 receptor in cancer and cancer gene therapy. Cancer Gene Ther. 2008; 15: 413-48.

30. Choi BK, Cai X, Yuan B, Huang Z, Fan X, Deng H, et al. HER3 intracellular domains play a crucial role in HER3/HER2 dimerization and activation of downstream signaling pathways. Protein Cell. 2012; 3: 781-9.

31. Ling B, Wang GX, Long G, Qiu JH, Hu ZL. Tumor suppressor miR-22 suppresses lung cancer cell progression through post-transcriptional regulation of ErbB3. J Cancer Res Clin Oncol. 2012; 138: 1355-61.

32. Wang $X$, Zhu X, Zhang $H$, Wei S, Chen $Y$, Chen $Y$, et al. Increased circular RNA hsa_circ_0012673 acts as a sponge of miR-22 to promote lung adenocarcinoma proliferation. Biochem Biophys Res Commun. 2018; 496: 1069-75.

33. Ma X, Yang X, Bao W, Li S, Liang S, Sun Y, et al. Circular RNA circMAN2B2 facilitates lung cancer cell proliferation and invasion via miR-1275/FOXK1 axis. Biochem Biophys Res Commun. 2018; 498: 1009-15.

34. Liu W, Ma W, Yuan Y, Zhang Y, Sun S. Circular RNA hsa_circRNA_103809 promotes lung cancer progression via facilitating ZNF121-dependent MYC expression by sequestering miR-4302. Biochem Biophys Res Commun. 2018; 500: 846-51.

35. Qu DH, Yan BD,Xin R,Ma TG. A novel circular RNA hsa circ 0020123 exerts oncogenic properties through suppression of miR-144 in non-small cell lung cancer. Am J Cancer Res. 2018; 8: 1387-402.

36. Jiang MM, Mai ZT, Wan SZ, Chi YM, Zhang X, Sun BH, et al. Microarray profiles reveal that circular RNA hsa_circ_0007385 functions as an oncogene in non-small cell lung cancer tumorigenesis. J Cancer Res Clin Oncol. 2018; 144: 667-74.

37. Zhao F, Han $Y$, Liu Z, Zhao Z, Li Z, Jia K. circFADS2 regulates lung cancer cells proliferation and invasion via acting as a sponge of miR-498. Biosci Rep. 2018; 38.

38. Tan S, Gou Q, Pu W, Guo C, Yang Y, Wu K, et al. Circular RNA F-circEA produced from EML4-ALK fusion gene as a novel liquid biopsy biomarker for non-small cell lung cancer. Cell Res. 2018; 28: 693-5.

39. Tan S, Sun D, Pu W, Gou Q, Guo C, Gong Y, et al. Circular RNA F-circEA-2a derived from EML4-ALK fusion gene promotes cell migration and invasion in non-small cell lung cancer. Mol Cancer. 2018; 17: 138 .

40. Guarnerio J, Bezzi M, Jeong JC, Paffenholz SV, Berry K, Naldini MM, et al. Oncogenic Role of Fusion-circRNAs Derived from Cancer-Associated Chromosomal Translocations. Cell. 2016; 165: 289-302.

41. Chang H, Qu J, Wang J, Liang X, Sun W. Circular RNA circ_0026134 regulates non-small cell lung cancer cell proliferation and invasion via sponging miR-1256 and miR-1287. Biomed Pharmacother. 2019; 112: 108743

42. Zhou $\mathrm{Y}$, Zheng X, Xu B, Chen L, Wang Q, Deng H, et al. Circular RNA hsa_circ_0004015 regulates the proliferation, invasion, and TKI drug resistance of non-small cell lung cancer by miR-1183/PDPK1 signaling pathway. Biochem Biophys Res Commun. 2019; 508: 527-35.

43. Xue YB, Ding MQ, Xue L, Luo JH. CircAGFG1 sponges miR-203 to promote EMT and metastasis of non-small-cell lung cancer by upregulating ZNF281 expression. Thorac Cancer. 2019; 10: 1692-701.

44. Tian F, Yu CT, Ye WD, Wang Q. Cinnamaldehyde induces cell apoptosis mediated by a novel circular RNA hsa circ 0043256 in non-small cell lung cancer. Biochem Biophys Res Commun. 2017; 493: 1260-6.

45. Dai X, Zhang N, Cheng Y, Yang T, Chen Y, Liu Z, et al. RNA-binding Protein Trinucleotide repeat-containing 6A Regulates the Formation of Circular RNA 0006916, with Important Functions in Lung Cancer Cells. Carcinogenesis. 2018

46. Zhang $\mathrm{Y}$, Zhao $\mathrm{H}$, Zhang L. Identification of the tumorsuppressive function of circular RNA FOXO3 in nonsmall cell lung cancer through sponging miR155. Mol Med Rep. 2018; 17: 7692-700.

47. Yang L, Wang J, Fan Y, Yu K, Jiao B, Su X. Hsa_circ_0046264 up-regulated BRCA2 to suppress lung cancer through targeting hsa-miR-1245. Respir Res. 2018; 19: 115

48. Huang MS, Liu JY, Xia XB, Liu YZ, Li X, Yin JY, et al. Hsa_circ_0001946 Inhibits Lung Cancer Progression and Mediates Cisplatin Sensitivity in Non-small Cell Lung Cancer via the Nucleotide Excision Repair Signaling Pathway. Front Oncol. 2019; 9: 508

49. Wei S, Zheng $Y$, Jiang $Y$, Li X, Geng J, Shen $Y$, et al. The circRNA circPTPRA suppresses epithelial-mesenchymal transitioning and metastasis of NSCLC cells by sponging miR-96-5p. EBioMedicine. 2019; 44: 182-93.

50. Zhao J, Li L, Wang Q, Han H, Zhan Q, Xu M. CircRNA Expression Profile in Early-Stage Lung Adenocarcinoma Patients. Cell Physiol Biochem. 2017; 44: 2138-46.

51. Luo YH, Zhu XZ, Huang KW, Zhang Q, Fan YX, Yan PW, et al. Emerging roles of circular RNA hsa_circ_0000064 in the proliferation and metastasis of lung cancer. Biomed Pharmacother. 2017; 96: 892-8.

52. Zong L, Sun $\mathrm{Q}$, Zhang $\mathrm{H}$, Chen $\mathrm{Z}$, Deng $\mathrm{Y}, \mathrm{Li} \mathrm{D}$, et al. Increased expression of circRNA_102231 in lung cancer and its clinical significance. Biomed Pharmacother. 2018; 102: 639-44.

53. Zhang S, Zeng X, Ding T, Guo L, Li Y, Ou S, et al. Microarray profile of circular RNAs identifies hsa_circ_0014130 as a new circular RNA biomarker in non-small cell lung cancer. Sci Rep. 2018; 8: 2878.

54. Gu X, Wang G, Shen H, Fei X. Hsa_circ_0033155: A potential novel biomarker for non-small cell lung cancer. Exp Ther Med. 2018; 16: 3220-6.

55. Liu XX, Yang YE, Liu X, Zhang MY, Li R, Yin YH, et al. A two-circular RNA signature as a noninvasive diagnostic biomarker for lung adenocarcinoma. J Transl Med. 2019; 17: 50.

56. Zhu X, Wang X, Wei S, Chen Y, Chen Y, Fan X, et al. hsa_circ_0013958: a circular RNA and potential novel biomarker for lung adenocarcinoma. FEBS J. 2017; 284: 2170-82.

57. Hang D, Zhou J, Qin N, Zhou W, Ma H, Jin G, et al. A novel plasma circular RNA circFARSA is a potential biomarker for non-small cell lung cancer. Cancer Med. 2018; 7: 2783-91.

58. WANG J, LI H. CircRNA circ_0067934 silencing inhibits the proliferation, migration and invasion of NSCLC cells and correlates with unfavorable prognosis in NSCLC. Eur Rev Med Pharmacolo Sci. 2018; 22: 3053-60.

59. Yao JT, Zhao SH, Liu QP, Lv MQ, Zhou DX, Liao ZJ, et al. Over-expression of CircRNA 100876 in non-small cell lung cancer and its prognostic value. Pathol Res Pract. 2017; 213: 453-6.

60. Li Y, Hu J, Li L, Cai S, Zhang H, Zhu X, et al. Upregulated circular RNA circ 0016760 indicates unfavorable prognosis in NSCLC and promotes cell progression through miR-1287/GAGE1 axis. Biochem Biophys Res Commun. 2018; 503: 2089-94

61. Jiali Xu YS, Tongpeng Xu, Wei Zhu, Tianzhu Qiu, Jun Li, Meiling Zhang, Jing $\mathrm{Xu}$, Renhua Guo, Kaihua Lu, Lingjun Zhu, Yongmei Yin, Yanhong $\mathrm{Gu}$, Lianke Liu, Ping Liu, Rong Wang. Microarray expression profiling and bioinformatics analysis of circular RNA expression in lung squamous cell carcinoma. Am J Transl Res. 2018; 3: 771-83.

62. Han J, Zhao G, Ma X, Dong Q, Zhang H, Wang Y, et al. CircRNA circ-BANP-mediated miR-503/LARP1 signaling contributes to lung cancer progression. Biochem Biophys Res Commun. 2018; 503: 2429-35. 
63. Li S, Sun X, Miao S, Lu T, Wang Y, Liu J, et al. hsa_circ_0000729, a potential prognostic biomarker in lung adenocarcinoma. Thorac Cancer. 2018; 9: 924-30.

64. Tian F, Wang Y, Xiao Z, Zhu X. Circular RNA CircHIPK3 Promotes NCI-H1299 and NCI-H2170 Cell Proliferation through miR-379 and its Target IGF1. Zhongguo Fei Ai Za Zhi. 2017; 20: 459-67.

65. Chen X, Mao R, Su W, Yang X, Geng Q, Guo C, et al. Circular RNA circHIPK3 modulates autophagy via MIR124-3p-STAT3-PRKAA/AMPKalpha signaling in STK11 mutant lung cancer. Autophagy. 2019: 1-13.

66. Yin Y, Gao H, Guo J, Gao Y. Effect of Circular RNA UBAP2 Silencing on Proliferation and Invasion of Human Lung Cancer A549 Cells and Its Mechanism. Zhongguo Fei Ai Za Zhi. 2017; 20: 800-7.

67. Ding L, Yao W, Lu J, Gong J, Zhang X. Upregulation of circ_001569 predicts poor prognosis and promotes cell proliferation in non-small cell lung cancer by regulating the Wnt/ $\beta$-catenin pathway. ONCOLOGY LETTERS. 2018; 16: 453-8.

68. Li J, Wang J, Chen Z, Chen Y, Jin M. Hsa circ 0079530 promotes cell proliferation and invasion in non-small cell lung cancer. Gene. 2018; 665: 1-5.

69. Liu G, Shi H, Deng L, Zheng H, Kong W, Wen X, et al. Circular RNA circ-FOXM1 facilitates cell progression as ceRNA to target PPDPF and MACC1 by sponging miR-1304-5p in non-small cell lung cancer. Biochem Biophys Res Commun. 2019; 513: 207-12. 